\title{
UTILIZZAZIONE, PER LA REGISTRAZIONE FOTOGRAFICA DEL POTENZIALE DEL CAMPO ELETTRICO ATMOSFERICO, DI UN ELETTROMETRO BENNDORF, IN ORIGINE A REGISTRAZIONE MECCANICA
}

\author{
Salvatore Gandolfo
}

1. Cenni informativi. -- Nel giugno del 1948 ebbi l'incarico da parte del Prof. A. Lo Surdo, allora Direttore dell'Istituto Nazionale di Geofisica, di studiare il regime di variazione diurna del potenziale del campo elettrico atmosferico, a Messina.

Per la registrazione fu destinato un elettrometro Benndorf $\left({ }^{1}\right),\left({ }^{2}\right)$, fuori uso, dell'Istituto Geofisico e Geodetico dell'Università della stessa città.

Mentre fu relativamente agevole rimettere in efficienza la parte elettrica dello strumento. invece il meccanismo ad orologeria ed il complesso registratore diedero luogo ad inconvenienti che, malgrado i ripetuti aggiustagri, non fu possibile eliminare in modo definitivo. Si perdette notevole tempo perché, in situ, non era allora possibile disporre, del materiale necessario per assicurare un buon isolamento ai quadranti e all'equipaggio mol,ile dell'elettrometro, e del filo adatto per la sospensione.

La dotazione finanziaria dell'Istituto, impegnato nella ricostruzione di tutto quanto era stato sottratto o distrutto, durante l'ultima guerra, non permettera di affrontare la spesa necessaria per l'acquisto di un altro elettrometro che potesse sostituire il Benndorf, né di apportare a questo le modifiche radicali indispensabili.

Solo con la assidua e snervante sorveglianza, e i frefuenti interventi per ovviare agli inconvenienti che si presentavano, si riusci, durante l'anno 1950, ad ottenere delle estese serie di registrazioni, che hanno fornito il materiale di studio per alcune ricerche, sulle quali si riferisce in altre note $\left({ }^{3}\right)$.

Diverse furono però le lacune subite dalle registrazioni, specie per il difettoso funzionamento del complesso registratore. 
Essendosi resa disponihile, nel 1951, una modesta somma da destinare per le ricerche di elettricitì atmosferica, tale però da non consentire l'acquisto di un nuovo strumento registratore, si decise di impiegarla per modificare e nello stesso tempo perfezionare l'apparecchiatura esistente.

2. Modifiche e perfezionamenti. - T'uso continuo per tutto l'anno 1950 dell'elettrometro Benndorf e le esigenze delle ricerche, in programma di esecuzione, suggerirono, per raggiungere l'optimum del suo funzionamento elettrico e la garanzia della continuitì delle registrazioni, di :

a) - alleggerire l'equipaggio mohile del peso costituito dal suo lungo indice metallico e dal contrappeso destinato ad equilibrarlo,

b) - assicurare la simmetria cilindrica all'equipaggio mobile, della quale l'ideatore dello strumento non aveva tenuto conto,

c) - eliminare il sistema di registrazione per battuta sull'indice dell'equipaggio mobile, per evitare di sottoporre, ogni due minuti, questo, la sua sospensione, e l'ammortizzatore, al tormento meccanico derivante dall'azione energica dell'elettrocalamita,

d) - eliminare il sistema dei rulli trattori della striscia di carta, messi in moto dall'orologio, perché davano luogo ad inceppamenti e quindi ad interruzioni delle registrazioni,

e) - realizzare la registrazione continua, per avere modo di seguire la microstruttura delle variazioni, non rilevabile mediante la registrazione per punti.

Tutto ciò poteva essere conseguito senza rinunciare al corpo dell'elettrometro, procedendo al semplice adattamento di esso alla registrazione fotografica, spogliandolo di tutte le parti che si rendevano inutili, con l'approntamento di un registratore.

Per rendere possibile, infatti, la registrazione fotografica si è fissato uno specchietto piano all'asse dell'equipaggio mobile dell'elettrometro, in modo tale da essere in grado di riflettere l'immagine di un sottile filamento luminoso, prodotta da un proiettore disposto lateralmente allo strumento.

Il registratore è stato costruito in modo notevolmente semplice e tale da richiedere una modica spesa.

Una fenditura, posta nella parte anteriore di esso, intercetta un piccolo segmento luminoso, che viene focalizzato da una lente cilin- 
drica, spostabile con movimento micrometrico su guide, in un puntino sulla carta sensibile avvolta sul tamburo, costituito da un cilindro, avente l'altezza di $\mathrm{cm}$. 26 ed il raggio di base $\mathrm{cm}$. 12.2.

Il movimento di questo è stato realizzato, su cuscinetti a sfere, per mezzo di un meccanismo ad orologeria, che faceva parte di un igrografo fuori uso, mettendolo in condizione di ruotare, anch'esso su cuscinetti a sfere, però intorno ad un asse orizzontale. (Nell'igrograto il meccanismo ad orologeria, ruotava insieme al tamburo, intorno ad un asse verticale, come avviene per la maggior parte dei registratori in uso nelle stazioni meteorologiche, e senza appoggio su cuscinetti a sferel.

Avvitando un dado zigrinato, posto esternamente su una delle fiancate del registratore, si può rendere solidale il meccanismo ad orologeria al tamburo; svitandolo, questi si possono liberare uno dall'altro.

Con la prima manovra il tamburo è costretto a girare insieme al meccanismo ad orologeria, e l'apparecchiatura è disposta per la registrazione, con la seconda manovra il tamburo è " in folle " e permette di eseguire, in luce rossa, agevolmente nel giro di qualche minuto, l'operazione giornaliera di cambio della carta sensibile.

Il tamburo esegue un giro completo nelle 24 ore, con scorrimento di poco magriore di $\mathrm{cm} .3$ all'ora.

La carica del meccanismo ad orologeria è tale che permette al tamburo di girare ininterrottamente per una settimana.

L'elettrometro ed il registratore sono protetti da una custodia, costruita in masonite, divisa in due parti, una delle quali riservata al primo e l'altra al secondo. Le due parti comunicano solo attraverso la fenditura del registratore.

3. Campi di misura. - Se, come si è praticato, a Messina, per alcune ricerche, in campo normale, si ha cura di portare lo zero dello strumento su una delle zone marginali della carta sensibile (avente le dimensioni di cm. $24 \times 76$ ), si possono registrare sino a +600 volts, con la sensibilità necessaria al tipo di ricerche che di solito si eseguono.

Nella fig. 1 è riprodotta una registrazione ottenuta, in condizioni meteorologiche di normalità, con l'elettrometro Benndorf originale.

Nella fig. 2 sono riportate tre registrazioni effettuate, in condizioni meteorologiche di normalita, con l'elettrometro Benndorf modificato.

Lo stesso elettrometro, qualora si agisca opportunamente sulla 


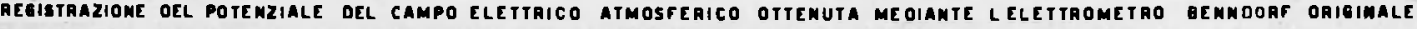

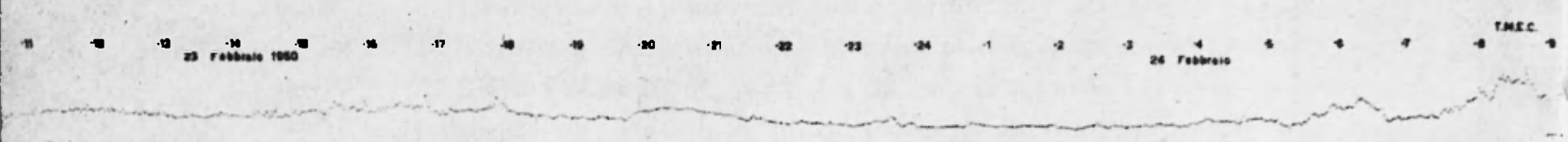

Fi6.1

Fig. 1

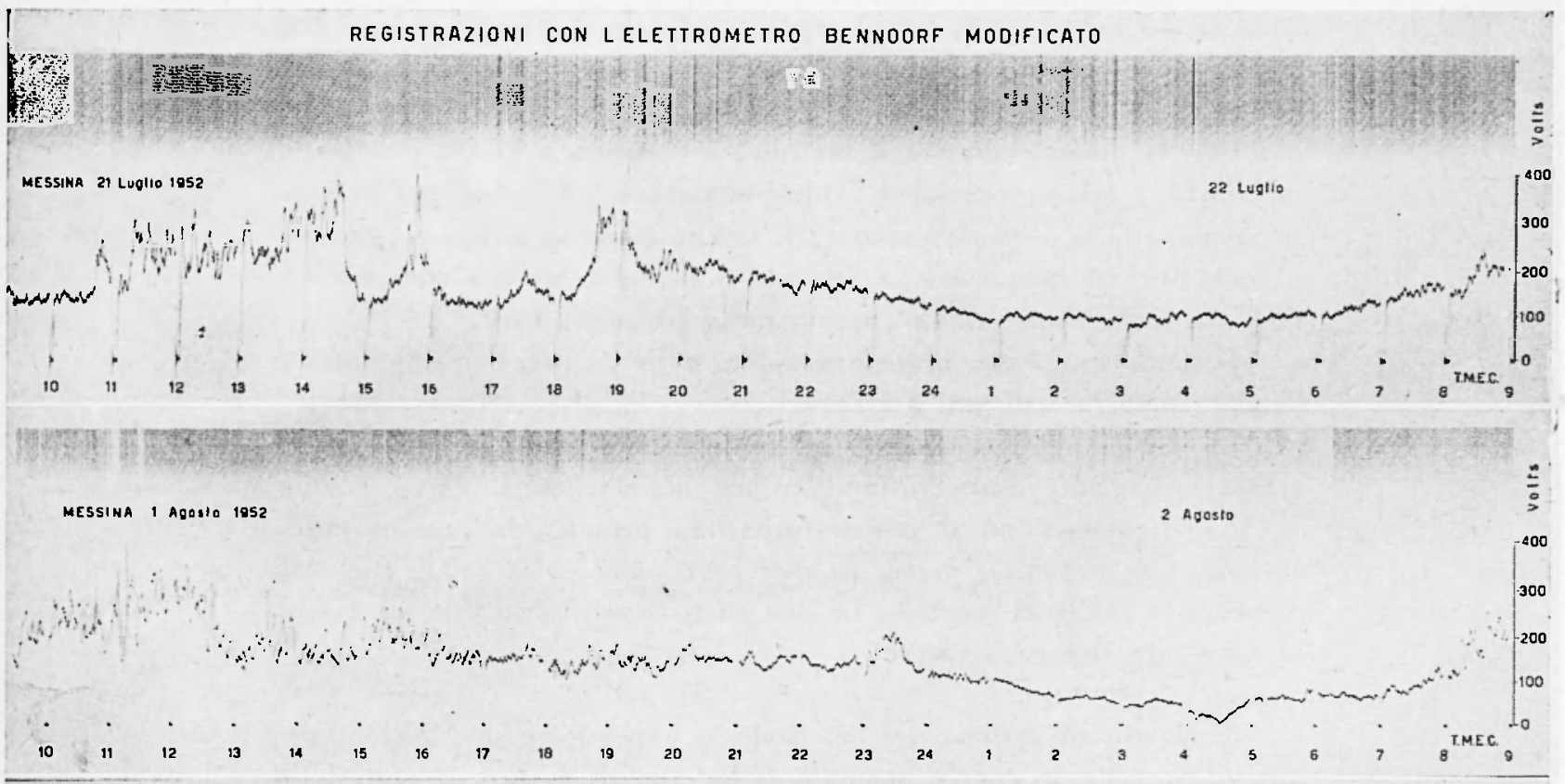

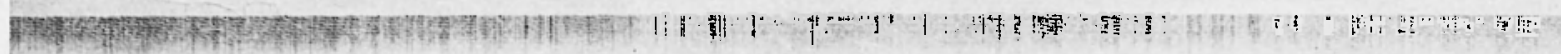

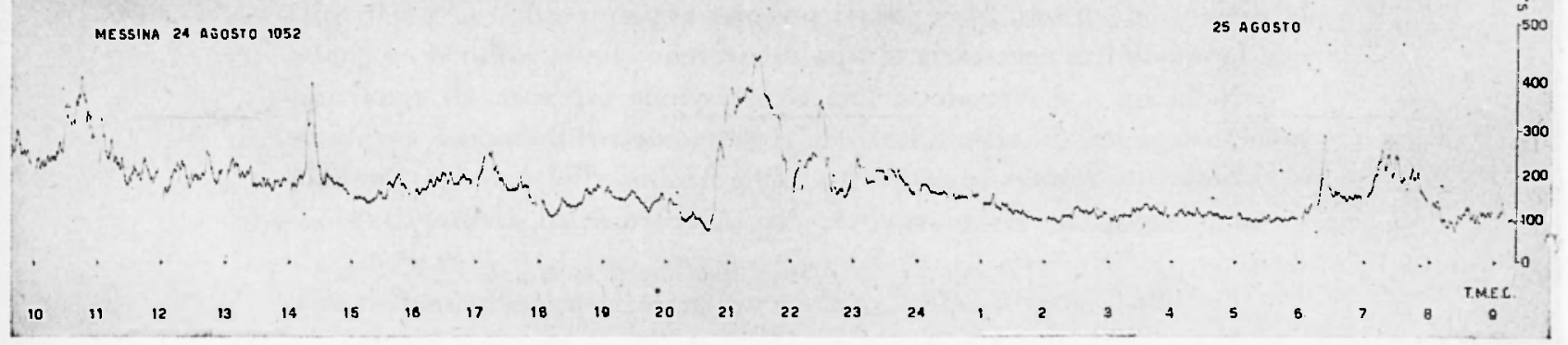

Fig. 2 
lunghezza e apertura della sospensione hifilare, e sulla tensione ai quadranti, si puo adibire per la registrazione degli elevati potenziali che si hanno in condizioni meteorologiche perturbate non temporalesche, come $\dot{c}$ stato accertato in occasione delle prove protrattesi per alcuni mesi, nella stagione invernale 1952-1953.

Nella fig. 3 sono riprodotte tre registrazioni ottenute in tali condizioni.

E da avrertire che la scala, riportata su uno dei margini delle registrazioni, e stata determinata sperimentalmente solo sino a 1000 volts, in quanto non si disponeva, né tutiora si dispone, delle tensioni magriori da fornire all'ago per la taratura.

Solo per avere una idea dell'ordine dei valori raggiunti, essa è stata estrapolata per potenziali maggiori (parte tratteggiata dell'asse delle ordinatel, ammettendo l'andamento teorico per gli angoli di rotazione dell'equipaggio molile dell'elettrometro, al variare della tensione assunta dallo stesso, tenuto conto che la registrazione avviene su punti appartenenti a generatrici di un cilindro.

Pur non essendo stato possibile eseguire uno studio quantitativo, le registrazioni ottenute hanno fornito, però, elementi che destano interesse nei riguardi delle singolarita che presenta il potenziale del campo elettrico atmosferico perturbato.

Su queste si spera riferire in altra nota, appena si disporrà di una sufficiente casistica.

1. Registrazione del tempo. - In campo normale, la registrazione del tempo, per dare la possibilita di un agevole spoglio dei diagrammi, si è realizzata su un margine della carta sensiloile, fig. 2, mediante segmenti soltili luminosi, generati da un apposito proiettore, inserito in un circuito elettrico che viene chiuso ogni due minuti dall'orologio che faceva parte del complesso Benndorf (*).

Ogni ora 1111 relais provvede a mettere in connessione con la terra l'ago dell'elettrometro, di guisa che, per alcuni minuti, resta segnato sulla carta sensibile il potenziale zero di riferimento, fig. 2 .

In campo perturbato, allo scopo di non influire interrompendo e fuindi deformando le singolariti nelle registrazioni, il segnale dellora si i realizzato al margine della carta, in modo simile a quello dei due minuti, fig. 3 .

(i) Recentemente, per rendere sempre più agevole lo spoglio delle registrazioni, le traccie relative agli intervalli di due minuti si sono realizzate per tutta la larghezza della striscia di carta sensibile. 


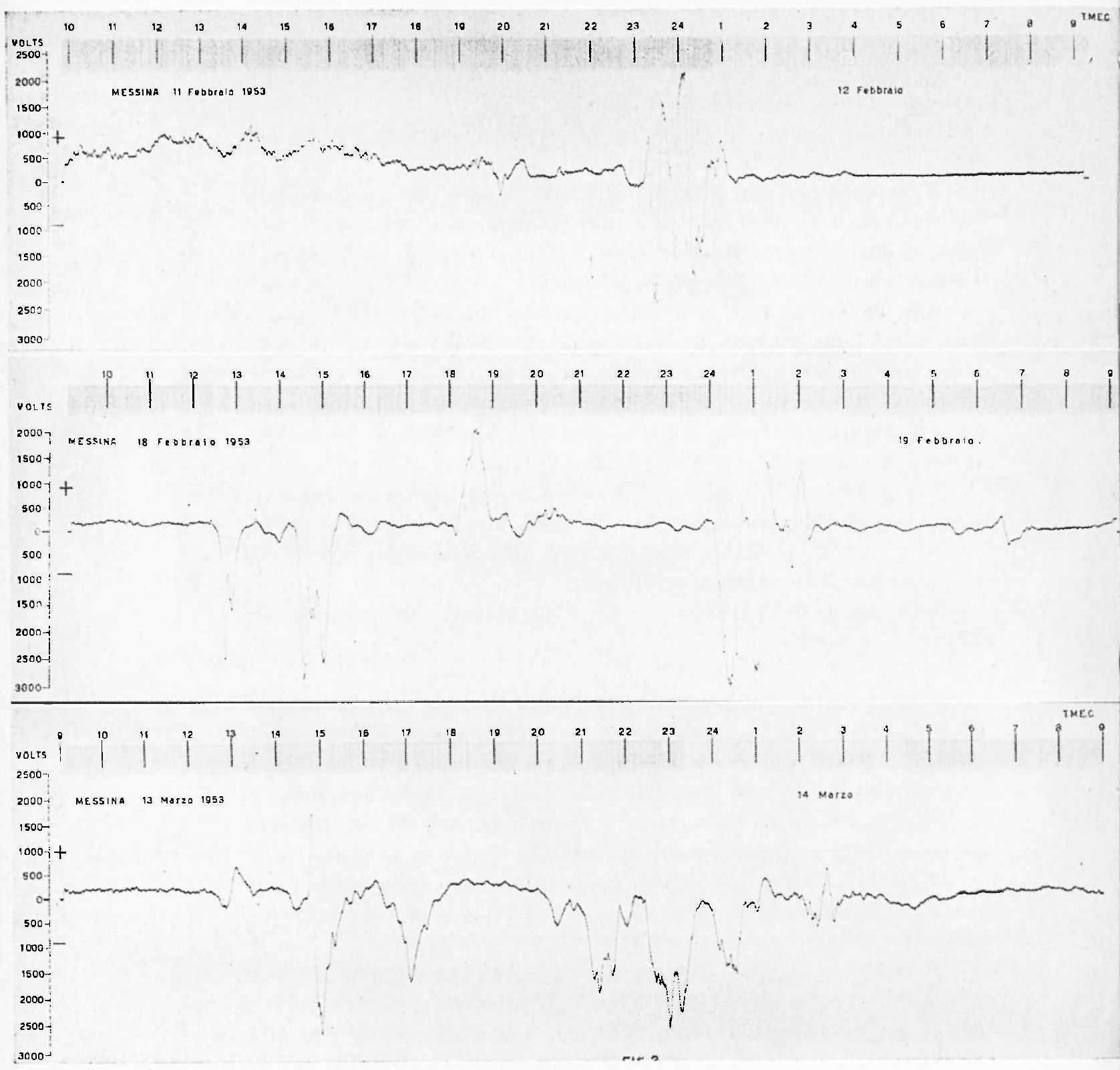

Fig. 3 
Il potenziale zero, in questo caso, viene segnato all'inizio e alla fine della registrazione.

5. Conclusioni. - I buoni risultati ottenuti, durante l'impiego continuo, per diversi mesi, senza alcun inconveniente notevole, dell'elettrometro e del registratore, di cui fanno fede le registrazioni, sia in campo normale che perturbato, riportate in questa nota, fig. 2,3 , consigliano, per la semplicità e costanza di buon funzionamento, l'adozione, nella misura del potenziale elettrico atmosferico, di apparecchiature simili a quella che, per la carenza dei mezzi disponibili, si è avuto occasione di sperimentare a Messina.

L'esperienza fatta ha dimostrato, tra l'altro, particolarmente interessante l'impiego del sistema sonda-elettrometro per la registrazione in campo perturbato non temporalesco.

Nella maggior parte degli osservatori, tuttora si registra il potenziale elettrico atmosferico in campo normale, e si perdono le indicazioni in campo perturbato, in quanto gli strumenti sono tarati con fondo scala di alcune centinaia di volts $\left({ }^{4}\right)$.

L'uso di due elettrometri del tipo già menzionato, opportunamente adattati e tarati, con registrazione su una stessa striscia di carta sensiluile, dì la possibilita di colmare, per buona parte, la lacuna lamentata, restando, per completare la registrazione del potenziale elettrico atmosferico, in campo perturlato, l'esigenza dell'uso di oscillografi a ragri catodici ("), per seguire le variazioni in occasione di temporali, durante i quali il sistema sonda-clettrometro non è idoneo.

Messina - Istituto Geofisico e Geodetico dell'Università - Osser. vatorio dell I.N.G. -.. Maggio 1954.

\section{RIASSUNTO}

Si riferisce sulle modifiche apportate ad un elettrometro Benndorf, in origine a registrazione meccanica, per realiziare, insieme ad un migliore funzionamento di esso. la registrazione fotografica del potensiale del campo elettrico atmosferico.

\section{SUMMARY}

Modifications have been carried out on a Benndorf electrometer originally having mechanical registration, together with some improvements in its functioning, in order to realize a photographic registration of the potential of the atmospheric electric field. 


\section{BIBLIOGRAFIA}

(1) H. Bexwdonf. Wiener Bericht III, 487, 1902.

(-) $) \quad$ Psysikalische Zeitschrift, 7, 98, 1906.

(3) S. Gandolfo, Annali di Geofisica, 1954, Roma (in corso di pubblicazione).

(t) J. A. Chalmers, Atmospheric Electricity, pag. 74, Oxford, 1949.

(5) H. Norinder, Annali di Geofisica, vol. II, n. 2, 1949. 